\title{
VECTOR-VALUED DISTRIBUTIONS AND THE SPECTRAL THEOREM FOR SELFADJOINT OPERATORS IN HILBERT SPACE
}

\author{
BY H. G. TILLMANN
}

Communicated by Edwin Hewitt, September 13, 1962

If $A$ is a bounded linear operator (with spectrum $S$ ) in a Banachspace $E$, the functional calculus of Dunford-Gelfand-Taylor ${ }^{1}$ can be characterized as a continuous homomorphism $f \rightarrow f(A)$ of the algebra $\mathfrak{H C}(S)^{2}$ of all holomorphic functions on $S$ into the Banach algebra $\mathcal{L}(E)$ with the properties $f(A)=I$ if $f(z) \equiv 1$ and $f(A)=A$ if $f(z) \equiv z$. If $E$ is a Hilbert space and $A=A^{*}$ a selfadjoint operator in $E$, there exist a much more general functional calculus $f \rightarrow f(A)=\int f(s) d E_{s}$, based on the spectral decomposition $A=\int s d E_{s}$ of $A$.

We will show how this extended calculus can be developed as a continuous extension of the analytic functional calculus in such a way, that we get a new and very natural proof of the spectral theorem for selfadjoint operators in Hilbert space.

1. The analytic functional calculus. If $S$ is the spectrum of $A$ the resolvent $R(s)=(A-s I)^{-1}$ is an operator valued, holomorphic function in the complement of $S$ on the Riemann-sphere $\Omega$, vanishing at $\infty$. If $f(z)$ is holomorphic on $S(:=$ holomorphic on a neighborhood of $S)$, there exists a contour $C$, separating $S$ from all singularities of $f$. Then

$$
f(A)=(2 \pi i)^{-1} \int_{C} f(s) R(s) d s=(2 \pi i)^{-1} \int_{C} f(s)(A-s I)^{-1} d s
$$

is independent of $C$ and $f \rightarrow f(A)$ is a homomorphism of the algebra $\mathfrak{H C}(S)$ of all such functions $f$ into the algebra $\mathscr{L}(E)$ of bounded endomorphisms of $E$.

This homomorphism is uniquely determined by the properties:

(a) $f(z) \equiv 1 \frown f(A)=I$,

(b) $f(z) \equiv z \sim f(A)=A$,

(c) $f_{n}(z) \Rightarrow f(z)$ (uniformly in a certain neighborhood of $S$ ) $f_{n}(A) \Rightarrow f(A)$ (in the norm topology of $\mathfrak{L}(E)$ ).

In certain cases the homomorphism can be extended to larger classes of functions. We will study the extension to the class of differentiable functions on the real line.

${ }^{1}$ See Dunford-Schwartz [1].

$2 \operatorname{HC}(S)$ is a topological algebra with the natural topology defined in Köthe [2]. 
2. Vector-valued distributions. ${ }^{3}$ A distribution $T$ on $\mathbf{R}$ with values in a locally convex vector space $F$ is a continuous linear mapping of the space $D=\mathcal{C}_{0}^{\infty}(\mathbf{R})$ into the space $F$.

We will assume that any Cauchy sequence in $F$ has a limit. If $T$ is a distribution with compact support (that means $T f=0$, if $f$ is identical zero in a neighborhood of a certain compact set $K \subset \mathbf{R}$ ), then $T$ can be extended in a unique way to a continuous linear mapping of the space $\varepsilon=\mathfrak{C}^{\infty}(\mathbf{R})$ into $F$.

The relation between boundary values of holomorphic functions and distributions (see $[5 ; 6 ; 7]$ ) can be generalized to the vectorvalued case, especially:

THEOREM 1. There exists a natural isomorphism of the space of Fvalued distributions $T$ with compact support and the space of $F$-valued holomorphic functions $\mathbf{t}(\cdot)$ in the complement of a compact set $K \subset \mathbf{R}$ with the property that $\mathrm{t}$ is also holomorphic at infinity and satisfies a condition ${ }^{4}$

$$
p(t(z)) \leqq M_{p}\left\{d(z, K)^{-m_{p}}+d(z, K)^{-1}\right\}
$$

for any continuous semi-norm $p$ in $F$.

This isomorphism is defined by the formulas

$$
\begin{aligned}
\mathbf{t}(z) & =(2 \pi i)^{-1} T_{s}\left[(s-z)^{-1}\right], \\
T f & =\lim _{\epsilon \rightarrow 0} \int f(s)\{\mathrm{t}(s+i \epsilon)-\mathrm{t}(s-i \epsilon)\} d s, \quad f \in D .
\end{aligned}
$$

3. The resolvent as indicatrix of an operator-valued distribution. We assume that the operator $A$ satisfies the following conditions:

(i) The spectrum of $A$ is contained in a compact set $K \subset \mathbf{R}$.

(ii) The resolvent $R(z)=(A-z I)^{-1}$ satisfies the condition

$$
\|R(z)\| \leqq M\left\{d(z, K)^{-m}+d(z, K)^{-1}\right\} .
$$

Then $(2 \pi i)^{-1} R(z)$ can be considered as the indicatrix of a distribution with support in $K$ and values in the Banach space $\mathscr{L}(E)$.

Theorem 2. If $A$ satisfies conditions (i) and (ii) then

(5) $T f=\lim _{\epsilon \rightarrow 0}(2 \pi i)^{-1} \int f(s)\{R(s+i \epsilon)-R(s-i \epsilon)\} d s=: f(A)^{5}$

defines a continuous homomorphism of the algebra $\mathfrak{C}^{\infty}(\mathbf{R})$ into $\mathfrak{L}(E)$ and

${ }^{3}$ See Schwartz [4].

$4(z, K)$ is the distance between $z$ and $K$.

6 The domain of integration may be any interval with $K$ in its interior. 
this is an extension of the homomorphism (1).

Conversely, if the homomorphism (1) can be extended to a continuous homomorphism of $\mathfrak{C}^{\infty}(\mathbf{R})$ into $\mathfrak{L}(E)$, then $A$ satisfies (i) and (ii).

An integration by parts shows that (5) is even defined for all functions which are differentiable a certain number of times: ${ }^{6}$

$$
\begin{aligned}
2 \pi i T f & =\lim _{\epsilon \rightarrow 0} \int f(s)\{R(s+i \epsilon)-R(s-i \epsilon)\} d s \\
& =\lim _{\epsilon \rightarrow 0}(-1)^{n} \int f^{(n)}(s)\left\{R^{(-n)}(s+i \epsilon)-R^{(-n)}(s-i \epsilon)\right\} d s \\
& =(-1)^{n} \int f^{(n)}(s)\left\{R^{(-n)}(s+i o)-R^{(-n)}(s-i o)\right\} d s, \quad n>m .
\end{aligned}
$$

This mapping is still multiplicative and we have a continuous homomorphism of the algebra $\mathfrak{C}^{n}(\mathbf{R})$ into $\mathscr{L}(E)$, if $n$ is greater than the constant $m$ in $\left(2^{\prime}\right)$.

4. Selfadjoint operators in Hilbert space. If $E=H$ is a Hilbert space, $A=A^{*}$ a selfadjoint, bounded operator in $H$, it is almost trivial that the conditions (i) and (ii) in $\$ 3$ are satisfied with constants $M=m=1$. From the resolvent equation

$$
R\left(s^{\prime}\right)-R(s)=\left(s^{\prime}-s\right) R\left(s^{\prime}\right) R(s)
$$

follows

$$
\begin{aligned}
f(A) & =T f=\lim (2 \pi i)^{-1} \int f(s)\{R(s+i \epsilon)-R(s-i \epsilon)\} d s \\
& =\lim \frac{\epsilon}{\pi} \int f(s) R(s+i \epsilon) R(s-i \epsilon) d s
\end{aligned}
$$

and for any vector $x \in H$ :

$$
\langle f(A) x, x\rangle=\lim \frac{\epsilon}{\pi} \int f(s)\|R(s-i \epsilon) x\|^{2} d s .
$$

The homomorphism $f \rightarrow f(A)$ of $\mathfrak{C}^{2}$ into $\mathfrak{L}(H)$ is positive: $f(s) \geqq 0$ on $\mathbf{R}$ implies $\langle f(A) x, x\rangle \geqq 0$ for all $x \in H$ and

$$
\|f(A)\| \leqq \sup _{\boldsymbol{s} \in \mathbf{R}}|f(s)| \text {. }
$$

This implies that the homomorphism $f \rightarrow f(A)$ can be extended by continuity (with respect to operator norms in $\mathscr{L}(H)$ ) to the class

${ }^{6}$ In (6) it is assumed that $f$ has compact support. But modification of $f$ outside of a neighborhood of $K$ does not change the value of $T f$. 
$\mathfrak{e}(\mathbf{R})$ of all continuous functions $f$.

Using the fact, that a bounded, monotonic sequence of operators in $H$ has a limit in the strong operator topology, one can construct a family of projections $E_{s}=\lim _{n} f_{s, n}(A)$, where $f_{s, n}$ is a suitable sequence of twice differentiable functions, converging monotonically to the characteristic function

$$
\chi_{s}(t)= \begin{cases}1 & \text { if } t<s, \\ 0 & \text { if } t \geqq s .\end{cases}
$$

It is easy to see that $\left\{E_{8}\right\}$ is a decomposition of the identity with the property that

$$
\int s d E_{\mathrm{s}}=\lim _{\Delta s \rightarrow 0} \sum s_{j}\left(E_{s_{j}}-E_{s_{j-1}}\right)=A .
$$

THEOREM 3. If $A$ is a selfadjoint, bounded linear operator in Hilbert space $H$, the homomorphism $f \rightarrow f(A)$ of (1) or (5) can be extended in a unique way (by continuity) to a continuous homomorphism of $\mathfrak{e}(\mathbf{R})$ into $\mathcal{L}(H)$ (with respect to the operator norm) and to a homomorphism of the algebra $\&$ of all Baire functions into $\mathbb{L}(H)$ (continuous with respect to monotonic convergence in $\mathbb{B}$ and strong convergence in $\mathcal{L}(H))$. This extension is given by

$$
f \rightarrow f(A)=\int f(s) d E_{\mathrm{s}}, \quad E_{\mathrm{s}}=\chi_{\mathrm{s}}(A)=\sup _{\boldsymbol{g} \leq \chi_{\mathrm{s}}} g(A), \quad g \in \mathbb{C}(\mathbf{R})
$$

$\left\{E_{s}\right\}$ is a decomposition of the identity with

$$
A=\int s d E_{8} .
$$

$\left\{E_{s}\right\}$ is uniquely determined by (14) and by $E_{s}=E_{s-0} . E_{s}$ commutes with every bounded operator that is commutable with $A$.

The last property follows immediately from the fact that $R(z)$ has this property of commutability, which is hereditary with respect to limits in the uniform and strong operator topology.

Complete proofs of these and some more general results will appear in $[8 ; 9]$.

\section{BiBLIOGRAPHY}

1. N. Dunford and J. T. Schwartz, Linear operators, Part I, Interscience, New York, 1958.

2. G. Köthe, Dualität in der Funktionentheorie, J. Reine Angew. Math. 191 (1953), 30-49. 
3. L. Schwartz, Theorie des distributions. I, II, Actualités Sci. Ind. Nos. 1091 and $1122=$ Publ. Inst. Math. Univ. Strasbourg, Hermann, Paris 1950/51.

4. - Theorie des distributions d valeurs vectorielles. I, Ann. Inst. Fourier (Grenoble) 7 (1957), 1-142.

5. H. G. Tillmann, Randverteilungen analytischer Funktionen und Distributionen, Math. Z. 59 (1953), 61-83.

6. - Distributionen als Randverteilungen analytischer Funktionen, Math. Z. 76 (1961), 5-21.

7. - Darstellung der Schwartzschen Distributionen durch analytische Funktionen, Math. Z. 77 (1961), 106-124.

8. - Darstellung der vektorwertigen Distributionen durch analytische Funktionen, Math. Ann. (to appear).

9. —- Eine Erweiterung des Funktionalkalkils fï lineare Operatoren, Math. Ann. (to appear).

UNIVERSITÄT HEIDELBERG AND

UNIVERSITY OF MARYLAND 